\title{
Effect of isotropic versus anisotropic elasticity on the electronic structure of cylindrical $\mathrm{InP} / \mathrm{In}_{0.49} \mathrm{Ga}_{0.51} \mathrm{P}$ self-assembled quantum dots
}

\author{
M. Tadić, ${ }^{*}$ F. M. Peeters, ${ }^{\dagger}$ and K. L. Janssens \\ Department of Physics, University of Antwerp (UIA), Universiteitsplein 1, B-2610 Antwerp, Belgium
}

(Received 15 October 2001; published 12 April 2002)

\begin{abstract}
The electronic structure of disk-shaped InP/InGaP self-assembled quantum dots is calculated within the effective-mass theory. The strain-dependent $6 \times 6$ multiband Hamiltonian for the valence band is simplified into an axially symmetric form. Both the continuum mechanical model, discretized by finite elements, and the isotropic model are used to calculate the strain distribution and their results are critically compared. The dependence of the electron and the hole energy levels on the dimensions of the quantum dot is investigated. We found that both the electron and hole energies are underestimated if the strain distribution is calculated by the isotropic elasticity theory. The agreement between the electron energies for the two approaches is better for thinner quantum dots. The heavy holes are confined inside the quantum dot, while the light holes are located outside the disk, but confined by the strain field near the edge of the disk periphery. We found that the $\hbar / 2$ hole ground state crosses the $3 \hbar / 2$ ground state when the height of the quantum dot increases and becomes the ground state for sufficiently thick quantum disks. The higher hole levels exhibit both crossings between the states of the different parity and anticrossings between the states of the same parity.
\end{abstract}

DOI: 10.1103/PhysRevB.65.165333

PACS number(s): 73.21.La, 62.20.Dc, 71.35.-y, 85.35.Be

\section{INTRODUCTION}

Recently, there has been an increasing interest ${ }^{1}$ in selfassembled quantum dots (SAQD's) in which carriers can be strongly confined. Various material combinations and dot shapes ${ }^{1}$ have been realized by the Stranski-Krastanow mode of epitaxial growth between lattice-mismatched semiconductors. The density, size, composition, and shape of the quantum dots are determined by the growth conditions (e.g., growth temperature, deposition time, flux, etc.). In view of this fact we will restrict ourselves to a model system, i.e., cylindrically shaped quantum $\operatorname{dot}^{2-4}$ in order to discuss trends in, e.g., the position of the electron and hole levels and mixing of the hole bands as function of the dimensions of the quantum dot. As an example, we consider the less-wellstudied $\mathrm{InP} / \mathrm{In}_{0.49} \mathrm{Ga}_{0.51} \mathrm{P}$ quantum dot system.

The dot and the semiconductor matrix the dot is incorporated in are made up of lattice-mismatched semiconductors, implying that large strain fields are present in SAQD's. In general, the distribution of the strain is inhomogeneous and anisotropic. Different theoretical calculation schemes exist to obtain the strain distribution in SAQD's. The continuum mechanical $(\mathrm{CM})$ model $^{5}$ has been recently compared with the valence force field (VFF) model in pyramidal quantum dots, ${ }^{6,7}$ and the two models were found to agree reasonably well. The main discrepancy exists near the dot boundary, where strong variations were found in the VFF results. The VFF method is, however, very slow and its accuracy depends on the employed interatomic model potential for the elastic energy. ${ }^{8}$ The simplest description of the strain distribution is achieved by the isotropic elasticity (IE) theory, which, as recently shown, ${ }^{9}$ reveals the main features produced by the CM model, but its usefulness for the electronic structure calculation has not been assessed as yet. The electron and the hole energy levels in self-assembled quantum dots are usually calculated by the effective-mass theory, which provides a transparent explanation for the experimental results and demands lower computational resources than more elaborate models. ${ }^{10}$ The complex three-dimensional multiband calculations have already been successfully applied to pyramidal ${ }^{5,7}$ and truncated pyramidal quantum dots. ${ }^{11}$ Even though strained cylindrical quantum dots have simpler geometry than pyramidal systems, the conduction band in them has only recently been analyzed. ${ }^{12}$ However, to the best of our knowledge, the electronic structure of the valence band in strained cylindrical quantum dots has not been explored. Provided the full axial symmetry of the Hamiltonian, the electronic structure may in principle be described by two spatial coordinates in these quantum dots.

In this paper, we calculate the strain distribution in diskshaped type-II InP/InGaP quantum dots using two different methods: the CM model and the IE theory. In the diskshaped quantum dots the IE approach is expected to work better than in pyramidal quantum dots. The discretization of the CM elastic energy functional is performed using firstorder finite elements. ${ }^{13}$ For the IE calculation, Davies' approach is adopted. ${ }^{9}$ The strain distribution modifies the electron and hole confinement potentials. The electronic structure of the valence band is computed using the multiband effective-mass theory but within the axially symmetric form. Recently, truncated pyramidal InP/InGaP quantum dots were studied theoretically, ${ }_{11}^{1}$ but the heavy and light hole projections of the valence band states were not resolved. In addition to the single-electron and -hole spectra, the dependence of the exciton transition energy on the height of the quantum dot for fixed radius of the disk is determined by a Hartreetype calculation. ${ }^{14,15}$ These energies are compared with the photoluminescence measurements on single quantum dots. ${ }^{2,3}$ This paper aims to provide a theoretical framework for the electronic structure of cylindrical quantum dots, to validate at a heuristic level the axial approximation for the valence band in strained quantum dots, and to assess the hole localization with the specific contributions of the heavy and light 


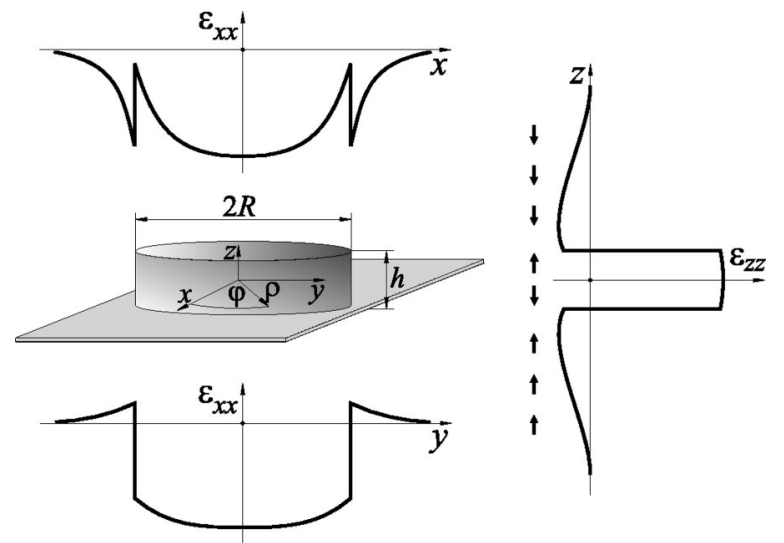

FIG. 1. A sketch of the cylindrical quantum dot. The CM calculated variations of $\varepsilon_{x x}$ along the $x$ and $y$ directions and of $\varepsilon_{z z}$ along the $z$ axis are shown. The direction of motion of the atoms in the $z$ direction is shown by the thick arrows. Cylindrical coordinates $\rho, \varphi$, and $z$ are also displayed. The wetting layer depicted as the shaded plane is discarded in the calculation.

holes to the valence band states. The devised model may also be employed for the fast estimation of the electronic structure in SAQD's having shapes similar to cylindrical, such as truncated conical or truncated hemispherical quantum dots.

The paper is organized as follows. In Sec. II, the calculation of the strain distribution is described. In Sec. III, the theory of the electronic structure is explained. In Sec. IV, the numerical results for the electronic structure are given and discussed.

\section{STRAIN CALCULATION}

The considered quantum disk and sketches of $\varepsilon_{x x}$ along $x$ and $y$, and $\varepsilon_{z z}$ along the $z$ direction, as calculated by the CM method, are depicted in Fig. 1. In reality, the cylindrical quantum dot is formed on a thin wetting layer, shown by the shaded plane in Fig. 1. It is well established that the wetting layer does not affect the strain distribution within SAQD's, ${ }^{6,16}$ and therefore is discarded in our calculation. As explicitly demonstrated below, $\varepsilon_{x x}$ is compressive in both the dot and the matrix, with sharp variations at the boundary in both the $x$ and $y$ direction. $\varepsilon_{z z}$ is tensile in the dot; its sign alters and exhibits a peak structure in the matrix.

To calculate the anisotropic strain distribution in our system we used the finite-element method. The elastic energy of our circular disk is given by ${ }^{17}$

$$
\begin{aligned}
E_{e l}= & \int_{V} d V\left[\frac{1}{2} C_{11}\left(\varepsilon_{x x}^{2}+\varepsilon_{y y}^{2}+\varepsilon_{z z}^{2}\right)\right. \\
& +C_{12}\left(\varepsilon_{x x} \varepsilon_{y y}+\varepsilon_{y y} \varepsilon_{z z}+\varepsilon_{z z} \varepsilon_{x x}\right) \\
& +2 C_{44}\left(\varepsilon_{x y}^{2}+\varepsilon_{y z}^{2}+\varepsilon_{z x}^{2}\right) \\
& \left.-2 \alpha\left(\varepsilon_{x x}+\varepsilon_{y y}+\varepsilon_{z z}\right) \varepsilon_{0}+3 \alpha \varepsilon_{0}^{2}\right] .
\end{aligned}
$$

Here $V$ denotes the equilibrium volume, $C_{11}, C_{12}$, and $C_{44}$ denote elastic moduli (elastic constants), $\varepsilon_{i j}$ is the $i j$ com- ponent of the strain tensor, $\alpha=C_{11} / 2+C_{12}$, and $\varepsilon_{0}$ denotes the misfit strain, which is zero in the barrier, while in the dot $\varepsilon_{0}=\left(a_{d}-a_{m}\right) / a_{m}$, where $a_{d}$ denotes the lattice constant in the dot and $a_{m}$ the lattice constant of the matrix semiconductor. The parameter $\alpha$ enforces the lattice mismatch between the two semiconductors. ${ }^{6}$ In our finite-element calculation the mesh consists of brick elements in the cylindrical coordinate system, shown in Fig. 1. The first-order ("hat") shape function, as it depends on the dimensionless coordinate $\xi$ defined on the interval $[-1,1]$ is defined by ${ }^{13}$

$$
f(\xi)= \begin{cases}(1+\xi) / 2, & -1 \leqslant \xi \leqslant 0 \\ (1-\xi) / 2, & 0<\xi \leqslant 1\end{cases}
$$

Products of these functions in all three directions are employed to discretize the spatial variation of the displacements on the elements. Positions of element vertices are denoted by $i j k$, where $i, j$, and $k$ correspond to the $\varphi, \rho$, and $z$ directions, respectively. The minimization of the elastic energy reduces to a system of linear equations: ${ }^{16}$

$$
\frac{d E_{e l}}{d u_{x(i j k)}}=\frac{d E_{e l}}{d u_{y(i j k)}}=\frac{d E_{e l}}{d u_{z(i j k)}}=0,
$$

where $u_{\xi(i j k)}$ represents the value of the $\xi(=x, y, z)$ component of the displacement vector at the $i j k$ vertex. In this approach, the right-hand side of the system of equations consists of volume integrals proportional to $\alpha$, which are conveniently modified by Green's theorem to surface integrals. ${ }^{13}$ Each vertex is coupled with 27 neighboring points, ${ }^{6}$ which when multiplied by the three components of the displacement vector gives a total of 81 nonzero coefficients in each equation. We applied a uniform mesh in the $\varphi$ direction and in the $\rho$ direction for $\rho<R$. In the $z$ direction and for $\rho>R$, a nonuniform mesh, with increasing density near the disk boundary is used. Due to the symmetry of the structure, only the first octant is relevant, and the normal components of the displacement are taken equal to zero at the symmetry planes. Furthermore, it is assumed that the structure completely relaxes far away from the dot. In other words all the displacement components are equal to zero at the surface of the discretization cylinder. The mesh comprises 33 vertices in the $\varphi$ direction and 65 in both the $\rho$ and $z$ directions, which gives a system of about $4 \times 10^{5}$ linear equations. As commented by Pryor et al., ${ }^{6}$ if the symmetric differences are employed in the functional relationships between strains and displacements, oscillatory solutions appear. In the finite-element approach, such oscillations are avoided. The system of linear equations is efficiently solved by the preconditioned conjugate gradient method.

Based on the ideas of Downes et al., ${ }^{18}$ Davies recently proposed an efficient method to calculate the strain distribution in an isotropic and homogeneous semiconductor. ${ }^{9}$ Starting from the analytical expression for the strain distribution around a spherical inclusion and using the analogy with electrostatics and applying the superposition principle, a Poisson equation was set up for a scalar potential from which the displacement vector is calculated as the gradient of the potential. By using the divergence theorem, Davies derived ${ }^{9}$ 
TABLE I. Experimental values of material parameters of InP and $\mathrm{In}_{0.49} \mathrm{Ga}_{0.51} \mathrm{P}$. All values, except the electron effective mass, are taken from Ref. 11. For the electron effective mass the value found in Ref. 31 is used.

\begin{tabular}{|c|c|c|}
\hline Parameter & InP & $\mathrm{In}_{0.49} \mathrm{Ga}_{0.51} \mathrm{P}$ \\
\hline$m_{e}\left(m_{0}\right)$ & 0.077 & 0.125 \\
\hline$\gamma_{1}$ & 4.95 & 5.24 \\
\hline$\gamma_{2}$ & 1.65 & 1.53 \\
\hline$\gamma_{3}$ & 2.35 & 2.21 \\
\hline$E_{g}(\mathrm{eV})$ & 1.424 & 1.97 \\
\hline$\Delta(\mathrm{eV})$ & 0.11 & 0.095 \\
\hline$a_{c}(\mathrm{eV})$ & -7.0 & -7.5 \\
\hline$a_{v}(\mathrm{eV})$ & 0.4 & 0.4 \\
\hline$b(\mathrm{eV})$ & -2.0 & -1.9 \\
\hline$\epsilon_{R}$ & 12.61 & 12.61 \\
\hline$C_{11}\left(10^{10} \mathrm{~N} / \mathrm{m}^{2}\right)$ & 10.22 & 12.17 \\
\hline$C_{12}\left(10^{10} \mathrm{~N} / \mathrm{m}^{2}\right)$ & 5.76 & 6.01 \\
\hline$C_{44}\left(10^{10} \mathrm{~N} / \mathrm{m}^{2}\right)$ & 4.6 & 5.82 \\
\hline$a(\mathrm{~nm})$ & 0.58687 & 0.56532 \\
\hline
\end{tabular}

$$
\varepsilon_{i j}(\mathbf{r})=\varepsilon_{0} \delta_{i j}-\frac{\varepsilon_{0}}{4 \pi} \frac{1+\nu}{1-\nu} \oint_{S^{\prime}} \frac{\left(r_{i}-r_{i}^{\prime}\right) d S_{j}^{\prime}}{\left|\mathbf{r}-\mathbf{r}^{\prime}\right|^{3}}
$$

Here, $\nu$ denotes the Poisson ratio, $S^{\prime}$ is the surface of the dot, and $r_{i}$ runs over $x, y$, and $z$. If the elastic constants $C_{11}, C_{12}$, and $C_{44}$ satisfy

$$
C_{11}-C_{12}-2 C_{44}=0,
$$

the strain distribution for the isotropic crystal is expected to be reproduced by the $\mathrm{CM}$ model. Moreover, we choose the Poisson ratio equal to $1 / 3$, which results in

$$
C_{12}=\frac{C_{11}}{2}, \quad C_{44}=\frac{C_{11}}{4} .
$$

$C_{12}$ and $C_{44}$ are computed for the given $C_{11}$ in InGaP (see Table I) and those elastic constants are also used for the InP dot. It may be shown that the average of the in-plane strain tensor components $\varepsilon_{a v}=\left(\varepsilon_{x x}+\varepsilon_{y y}\right) / 2$ depends only on the displacement along the radial coordinate: ${ }^{.7}$

$$
\varepsilon_{a v}=\frac{1}{2}\left(\varepsilon_{\rho \rho}+\varepsilon_{\varphi \varphi}\right)=\frac{1}{2} \frac{1}{\rho} \frac{\partial}{\partial \rho}\left(\rho u_{\rho}\right) .
$$

In elastically isotropic structures, neither $u_{\rho}$ nor $u_{z}$ depend on the polar angle; thus the distributions of both $\varepsilon_{a v}$ and $\varepsilon_{z z}$ along $\varphi$ are flat for fixed $\rho$ and $z$, hence the calculation of these strains, as needed for our axially symmetric electronic structure calculation, may be simplified to only these two coordinates.

In addition to anisotropy, the CM model may straightforwardly take into account the difference between the elastic constants in the dot and the matrix. But the IE theory assumes homogeneous elastic properties. ${ }^{9}$ In order to separate the influence of the anisotropy from the influence of the elastic constants on the electronic structure, the CM calculation
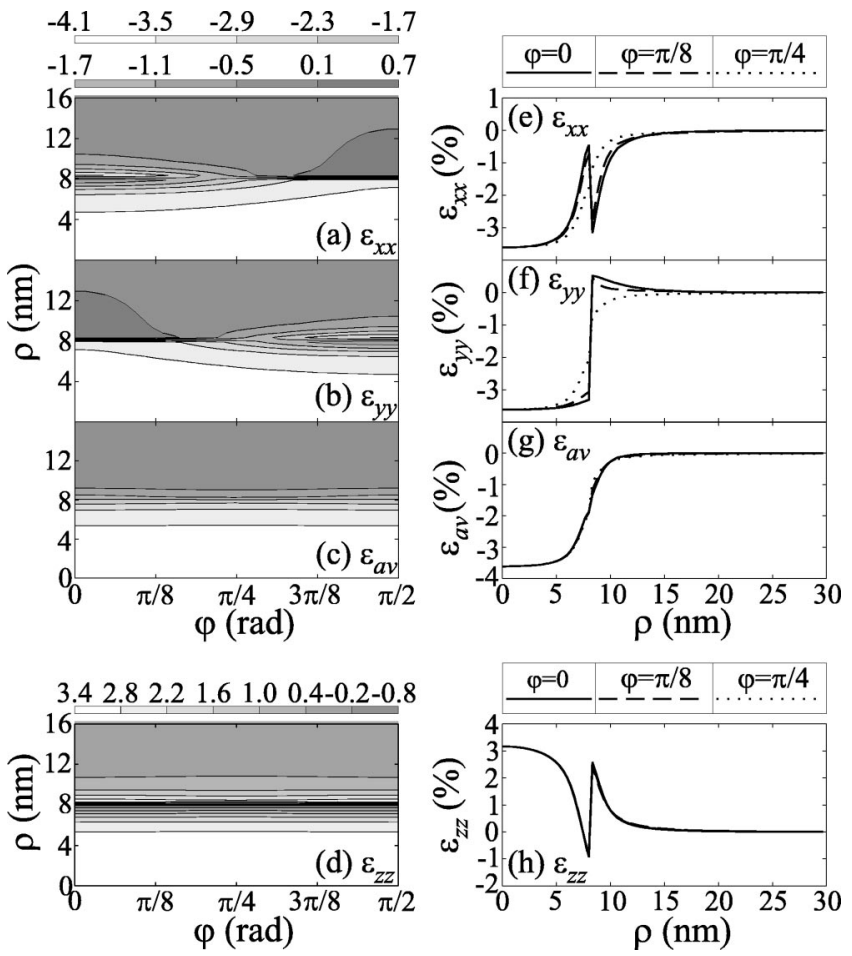

FIG. 2. Contour plot of the strain tensor components in the $z$ $=0$ plane: (a) $\varepsilon_{x x}$, (b) $\varepsilon_{y y}$, (c) $\varepsilon_{a v}=\left(\varepsilon_{x x}+\varepsilon_{y y}\right) / 2$, and (d) $\varepsilon_{z z}$. Cuts of these contour plots along $\rho$ for three different values of the polar angle $\varphi$ are shown in the right figures (e)-(h). The distributions of $\varepsilon_{a v}$ and $\varepsilon_{z z}$ shown in (g) and (h) almost coincide for the three chosen polar angles.

is also performed for the case of equal elastic constants in the dot and the barrier. The electronic structure calculated for these strains is hereafter denoted by $\mathrm{CM}^{0}$.

Contour plots of the diagonal components of the strain tensor and $\varepsilon_{a v}$ for a quantum disk with height $h=2.5 \mathrm{~nm}$ and radius $R=8 \mathrm{~nm}$ calculated by the CM model are shown in Figs. 2(a)-2(d), and cuts of these strain distributions along $\varphi=0$ ([100] direction), $\varphi=\pi / 8$, and $\varphi=\pi / 4$ ([110] direction) in the $z=0$ plane are displayed in Figs. 2(e) $-2(\mathrm{~h})$. Due to the cylindrical symmetry of the dot, $\varepsilon_{x x}$ is mirror symmetric around $\varphi=\pi / 4$ with respect to $\varepsilon_{y y}$. Figures 2(a), 2(b), 2(e), and 2(f) show that neither $\varepsilon_{x x}$ nor $\varepsilon_{y y}$ exhibit axial symmetry which would have resulted in all contour lines parallel to the $\varphi$ axis. $\varepsilon_{x x}$ is oscillatory near $\varphi=0$; it slowly increases inside the quantum dot and exhibits a positive bump near the dot boundary at $\varphi=\pi / 2$. Nevertheless, both the average of the two in-plane strain components, $\varepsilon_{a v}$ $=\left(\varepsilon_{x x}+\varepsilon_{y y}\right) / 2$, and $\varepsilon_{z z}$ are nearly axially symmetric, as displayed in Figs. 2(c) and 2(d), respectively, and also demonstrated by almost coinciding curves for the three chosen directions in Figs. 2(g) and 2(h), respectively. Referring to Figs. 2(a), 2(b), 2(e), and 2(f), one may conclude from a first glance that $\varepsilon_{x x}$ and $\varepsilon_{y y}$ differ substantially near $(x, y)$ $=(0, \pm R)$ and $(x, y)=( \pm R, 0)$. A more thorough inspection shows that the difference $\varepsilon_{x x}-\varepsilon_{y y}$ has peaks near the dot boundary, while in other parts of the structure it is almost negligible.

Since $\varepsilon_{a v}$ and $\varepsilon_{z z}$ are symmetric around the $z=0$ plane, 


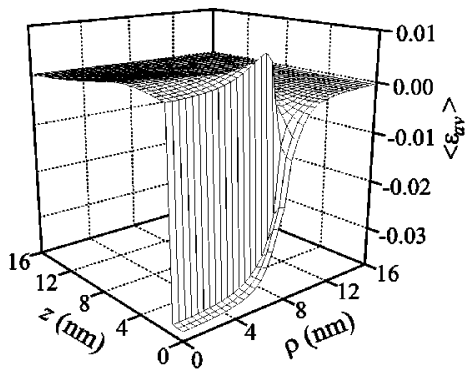

(a) $\mathrm{CM}$

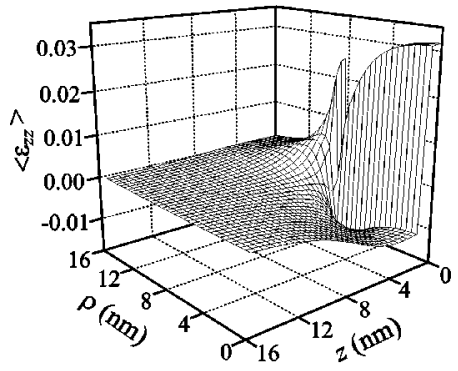

(b) $\mathrm{CM}$

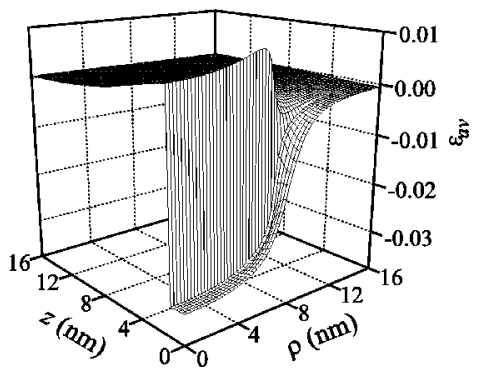

(c) IE

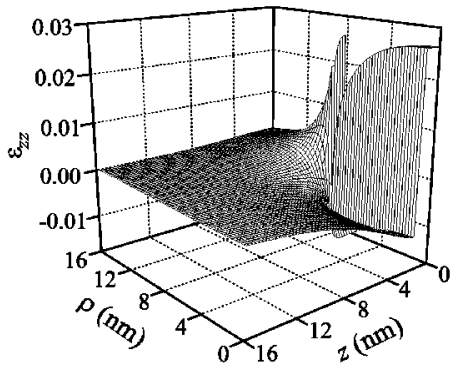

(d) IE
FIG. 3. The distribution of the strain components and their angular averages calculated from the two different strain models. (a) $\left\langle\varepsilon_{a v}\right\rangle$ calculated by the CM model. (b) $\left\langle\varepsilon_{z z}\right\rangle$ calculated by the CM model. (c) $\varepsilon_{a v}$ calculated by the IE theory. (d) $\varepsilon_{z z}$ calculated by the IE theory. the results of the IE and CM calculations for those strains are displayed in Figs. 3(a)-3(d) only for $z \geqslant 0$. The subsequent electronic structure calculation presented in Sec. III relies on $\varepsilon_{a v}$ and $\varepsilon_{z z}$ averaged over $\varphi$ :

$$
\left\langle\varepsilon_{a v}\right\rangle=\frac{1}{2 \pi} \int_{0}^{2 \pi} \varepsilon_{a v} d \varphi
$$

and

$$
\left\langle\varepsilon_{z z}\right\rangle=\frac{1}{2 \pi} \int_{0}^{2 \pi} \varepsilon_{z z} d \varphi .
$$

The IE results for $\varepsilon_{a v}$ and $\varepsilon_{z z}$, which do not depend on $\varphi$ as explained above, are shown in Figs. 3(c) and 3(d). Figures 3(a)-3(d) show the strain distributions for 2.5-nm-high quantum dots, but the same shapes are found for other quantum dot heights. One may notice the qualitative similarity of the strains calculated by the two methods. $\varepsilon_{a v}\left(\left\langle\varepsilon_{a v}\right\rangle\right)$ is compressive inside the quantum disk, but tensile in the barrier material. On the contrary, $\varepsilon_{z z}\left(\left\langle\varepsilon_{z z}\right\rangle\right)$ is tensile in the dot and compressive in the barrier. Both $\varepsilon_{a v}$ and $\left\langle\varepsilon_{a v}\right\rangle$ slowly increase along the $\rho$ direction, while both $\varepsilon_{z z}$ and $\left\langle\varepsilon_{z z}\right\rangle$ exhibit sharp discontinuities at $\rho=R$. A slight increase of $\left\langle\varepsilon_{a v}\right\rangle$ in the matrix along the $z$ axis near the center of the quantum dot, as shown in Fig. 3(a), is not present in the IE result for $\varepsilon_{a v}$ as shown in Fig. 3(c). In the latter case, the strain exhibits a steady decay along the $z$ axis. The difference between the two approaches is also demonstrated in the calculated $\varepsilon_{z z}$, where a monotonic decrease of the strain along the $z$ direction in Fig. 3(d) is replaced by a small peak structure in Fig. 3(b). However, both approaches indicate that the diagonal components of the strain tensor propagate a substantially larger distance along the $z$ direction than along the $\rho$ direction.

\section{ELECTRONIC STRUCTURE CALCULATION}

Mixing between the conduction and the valence band states due to both $\mathbf{k} \cdot \mathbf{p}$ and strain terms should be taken into account within the $8 \times 8$ or more complex multiband effective-mass models. ${ }^{19-21}$ It has been well established that the $\mathbf{k} \cdot \mathbf{p}$ coupling terms between the conduction and valence bands depend on the strain, ${ }^{19}$ and the proper treatment of the nanostructures based on low-band-gap semiconductors (such as InAs) should take these effects into account. ${ }^{20}$ However, in $\mathrm{InP} / \mathrm{InGaP}$ quantum dots both semiconductors have large band gaps, which implies that the mixing between the three topmost valence bands and the conduction band can be neglected without substantial loss of accuracy. Hence, the energy levels originating from the $\Gamma_{6}$ point in the cylindrical InP/InGaP quantum dots are extracted from the single-band Hamiltonian

$$
\begin{aligned}
H_{c}= & -\frac{\hbar^{2}}{2}\left[\left(\frac{1}{m_{e}}\right) \frac{\partial^{2}}{\partial z^{2}}+\frac{\partial}{\partial z}\left(\frac{1}{m_{e}}\right) \frac{\partial}{\partial z}\right] \\
& -\frac{\hbar^{2}}{2}\left\{\left(\frac{1}{m_{e}}\right)\left[\frac{\partial^{2}}{\partial \rho^{2}}+\frac{1}{\rho} \frac{\partial}{\partial \rho}+\frac{1}{\rho^{2}} \frac{\partial^{2}}{\partial \varphi^{2}}\right]\right. \\
& \left.+\frac{\partial}{\partial \rho}\left(\frac{1}{m_{e}}\right) \frac{\partial}{\partial \rho}\right\}+a_{c}\left\langle\varepsilon_{h y d}\right\rangle(\rho, z)+V_{c}(\rho, z),
\end{aligned}
$$

where $\varphi, \rho$, and $z$ denote the coordinates of the cylindrical system, $m_{e}$ is the electron effective mass, and $a_{c}$ is the hydrostatic deformation potential for the conduction band. The potential $V_{c}(\rho, z)=E_{c d}$ for $(\rho<R,|z|<h)$ and $E_{c m}$, otherwise, is due to the conduction band offset between the dot and the matrix. For cylindrical quantum dots, whose shape lacks sharp features, the piezoelectric potential is expected to be small and to alter the energy levels only in the order of 
$\mathrm{meV}^{22}$; hence, it is neglected in our calculation. Related to the small in-plane anisotropy of $\varepsilon_{a v}$ and $\varepsilon_{z z}$ discussed in Sec. II, the angular average of the hydrostatic strain $\left\langle\varepsilon_{\text {hyd }}\right\rangle$ is employed in the calculation of the electron energy levels. For such axially symmetric effective potential, the envelope angular quantum number $l_{e}$ is a good quantum number.

For the valence bands, the $\left\{\Gamma_{8}, \Gamma_{7}\right\}$ states are described by the $6 \times 6$ multiband Hamiltonian

$$
H_{v}=H_{k}+H_{s}+V_{v},
$$

where $H_{k}$ denotes the kinetic part, $H_{s}$ the strain-dependent part, and $V_{v}$ is the diagonal matrix containing the potentials for heavy holes, light holes, and the holes in the split-off band. The offset of the heavy and the light hole bands is $-45 \mathrm{meV} .{ }^{11}$ Both the kinetic and the strain-dependent part have the same form

$$
=\left[\begin{array}{cccccc}
E_{h h} & \sqrt{2} S & -S & 0 & -R & -\sqrt{2} R \\
\sqrt{2} S^{\dagger} & E_{l h} & \sqrt{2} Q & R & 0 & \sqrt{3} S \\
-S^{\dagger} & \sqrt{2} Q & E_{s o} & \sqrt{2} R & -\sqrt{3} S & 0 \\
0 & R^{\dagger} & \sqrt{2} R^{\dagger} & E_{h h} & \sqrt{2} S^{\dagger} & -S^{\dagger} \\
-R^{\dagger} & 0 & -\sqrt{3} S^{\dagger} & \sqrt{2} S & E_{l h} & \sqrt{2} Q \\
-\sqrt{2} R^{\dagger} & \sqrt{3} S^{\dagger} & 0 & -S & \sqrt{2} Q & E_{s o}
\end{array}\right] .
$$

The matrix elements of the kinetic part, labeled by the subscript $k$, are given by

$$
\begin{gathered}
E_{h h k}=P_{k}+Q_{k}, \quad E_{l h k}=P_{k}-Q_{k}, \quad E_{\text {sok }}=P_{k}+\Delta, \\
P_{k}=-\frac{\hbar^{2}}{2 m_{0}} \gamma_{1}\left(k_{x}^{2}+k_{y}^{2}+k_{z}^{2}\right) \\
Q_{k}=-\frac{\hbar^{2}}{2 m_{0}} \gamma_{2}\left(k_{x}^{2}+k_{y}^{2}-2 k_{z}^{2}\right) \\
R_{k}=\frac{\hbar^{2}}{2 m_{0}} \sqrt{3}\left[\frac{\gamma_{2}+\gamma_{3}}{2} k_{-}^{2}+\frac{\gamma_{2}-\gamma_{3}}{2} k_{+}^{2}\right] \\
S_{k}=\frac{\hbar^{2}}{2 m_{0}} \sqrt{6} \gamma_{3} k_{-} k_{z} .
\end{gathered}
$$

Here $k_{x}, k_{y}$, and $k_{z}$ denote the components of the wave vector along the crystallographic directions [100], [010], and [001], respectively, $k_{ \pm}=k_{x} \pm i k_{y}, \gamma_{1}, \gamma_{2}$, and $\gamma_{3}$ are the Luttinger parameters, and $\Delta$ is the spin-orbit split-off energy. The matrix elements of the strain-dependent Hamiltonian, all having the subscript $s$, are given by

$$
\begin{gathered}
E_{h h s}=P_{s}+Q_{s}, \quad E_{l h s}=P_{s}-Q_{s}, \quad E_{s o s}=P_{s}, \\
P_{s}=a_{v}\left(2 \varepsilon_{a v}+\varepsilon_{z z}\right), \quad Q_{s}=b\left(\varepsilon_{a v}-\varepsilon_{z z}\right),
\end{gathered}
$$

$$
\begin{gathered}
R_{s}=-\frac{\sqrt{3}}{2} b\left(\varepsilon_{x x}-\varepsilon_{y y}\right)+i d \varepsilon_{x y}, \\
S_{s}=-\frac{d}{\sqrt{2}}\left(\varepsilon_{z x}-i \varepsilon_{y z}\right),
\end{gathered}
$$

where $\varepsilon_{i j}$ denotes the $i j$ component of the strain tensor, and $a_{v}, b$, and $d$ are the deformation potentials for the valence band. The Hamiltonian given by Eq. (12) is written in the basis of periodic parts of Bloch functions in the center of the Brillouin zone $|J, j\rangle$, denoted by the angular quantum number $J$ and its projection on the $z$ axis $j$ :

$$
|3 / 2,3 / 2\rangle=\frac{i}{\sqrt{2}}|(X+i Y) \uparrow\rangle,
$$

$$
\begin{gathered}
|3 / 2,1 / 2\rangle=\frac{i}{\sqrt{6}}[|(X+i Y) \downarrow\rangle-2|Z \uparrow\rangle], \\
|1 / 2,1 / 2\rangle=\frac{i}{\sqrt{3}}[|(X+i Y) \downarrow\rangle+|Z \uparrow\rangle], \\
|3 / 2,-3 / 2\rangle=-\frac{i}{\sqrt{2}}|(X-i Y) \downarrow\rangle, \\
|3 / 2,-1 / 2\rangle=\frac{i}{\sqrt{6}}[|(X-i Y) \uparrow\rangle+2|Z \downarrow\rangle],
\end{gathered}
$$

$$
|1 / 2,-1 / 2\rangle=\frac{i}{\sqrt{3}}[|(X-i Y) \uparrow\rangle-|Z \downarrow\rangle] .
$$

Aside from being simpler, the $6 \times 6$ Hamiltonian used here does not contain the cumbersome strain derivatives, existing in the more comprehensive $8 \times 8$ model. $^{21}$ The in-plane warping of the constant energy surfaces depends on the square of $\left(\gamma_{3}-\gamma_{2}\right) / \gamma_{1},{ }^{23}$ which amounts to $14.1 \%$ and $13.0 \%$, in InP and InGaP, respectively, implying that only a small deviation of the band structure from axial symmetry is expected, and therefore the term proportional to $\gamma_{3}-\gamma_{2}$ in the $R_{k}$ matrix element, Eq. (13d), is neglected. Such axial approximation supplemented with the block-diagonalization ${ }^{24}$ of the multiband Hamiltonian is successfully applied to both unstrained and strained quantum wells, ${ }^{25,26}$ where the strain exists only in the well and is homogeneously distributed there. Recently, it has been applied to the unstrained quantum dots with parabolic in-plane confinement potential. ${ }^{23}$ For thin strained cylindrical quantum dots, this approximation requires further justification. As a matter of fact, we found that the shear strains are small in the whole structure, except near the dot boundary; therefore, we neglected the corresponding terms in Eqs. (14c) and (14d). Being small as discussed above, the in-plane anisotropy of $\varepsilon_{a v}$ and $\varepsilon_{z z}$ is neglected in our calculation by averaging the diagonal strain components over $\varphi$. Furthermore, since the difference $\varepsilon_{x x}-\varepsilon_{y y}$ is appreciable only in the vi- 
cinity of the dot boundary, we neglect it in Eq. (14c). Along with the assumed cylindrical shape of the quantum dot, all these approximations make up the axially symmetric multiband Hamiltonian, which has the form as given in Eq. (12), with

$$
\begin{gathered}
R_{k}=\frac{\hbar^{2}}{2 m_{0}} \sqrt{3} \frac{\gamma_{2}+\gamma_{3}}{2} k_{-}^{2}, \\
R_{s}=0, \quad S_{s}=0,
\end{gathered}
$$

while $\varepsilon_{a v}$ and $\varepsilon_{z z}$ are respectively replaced by $\left\langle\varepsilon_{a v}\right\rangle$ and $\left\langle\varepsilon_{z z}\right\rangle$ in Eq. (14b). The form of the other matrix elements, given by Eqs. (13a) $-(13 \mathrm{c})$ and (13e), is not modified, since they do not break the axial symmetry. The formed axially symmetric Hamiltonian for the holes commutes with the $z$ component of the total angular momentum $F_{z}=f \hbar$, composed of the Bloch part $J_{z}=j \hbar$ and the envelope part $L_{z}$ $=l \hbar$, i.e., $F_{z}=J_{z}+L_{z}$. This Hamiltonian is converted to cylindrical coordinates by noting that the kinetic part depends only on $k_{ \pm}, k_{z}$, and their products. $k_{ \pm}$expressed through $\rho$ and $z$ has the form

$$
k_{ \pm}=-i e^{ \pm i \varphi}\left(\frac{\partial}{\partial \rho} \pm \frac{i}{\rho} \frac{\partial}{\partial \varphi}\right) .
$$

Since $k_{x}$ and $k_{y}$ commute, we have $k_{x}^{2}+k_{y}^{2}=k_{+} k_{-}$.

Andreani et al. included the parity into the description of the $\Gamma_{8}$ valence band states in symmetric quantum wells. ${ }^{27}$ Edwards et al. subsequently extended this treatment to 6 $\times 6$ Luttinger-Kohn Hamiltonian, ${ }^{28}$ and Sercel and Vahala employed the same approach to describe the valence band states in quantum wires and dots. ${ }^{29}$ Ordering of the even and odd hole states depends on the width of the quantum well or the period of the superlattice, the effective confinement potentials, and effective masses of holes in different bands. Due to the action of the off-diagonal matrix elements of the Hamiltonian, the ordering pattern determined in the center of the two-dimensional Brillouin zone does not vary with the in-plane momentum. In other words, band mixing suppresses crossings between different subbands in quantum well structures.

In quantum dots, the transversal symmetry is removed by the finite and spatially variable potential in the plane of the quantum dot layer, and a range of in-plane wave vectors contribute to the quantum dot state. However, if the quantum dot is symmetric in the $z$ direction, parity of the wave function may be introduced as a good quantum number. The ordering of the even and odd states depends on the dimensions of the quantum dot, and crossings between the states of different parity may occur. ${ }^{23}$ Due to the neglect of the wetting layer, a reflection symmetry around the plane $z=0$ is recovered in our cylindrically symmetric quantum dots. From the structure of the multiband effective-mass Hamiltonian, we may easily determine that the even states in the valence band have the form

$$
F_{+}=\left[F_{h h+}, F_{l h-}, F_{s o-}, F_{h h-}, F_{l h+}, F_{s o+}\right]^{T},
$$

while the parity of the envelope functions in the hole spinor is reversed for the case of the odd hole states. Here $h h$ denotes the heavy hole, $l h$ the light hole, and so the spin-orbit split-off band. The eigenvalues for the given quantum number $f$ are denoted by $n X_{f}^{\sigma}$, where $n$ is the label of the state for the given $f, X$ denotes the minimum value of $|l|$ in the chosen basis set for the valence band states, and $\sigma$ is the parity of the state in the $z$ direction: + for even-parity states and for odd-parity states. The contribution of each band to the probability density $P$ is a sum over the two spin states, e.g., $P_{h h}=\Sigma_{i=1}^{2}\left|F_{h h i}\right|^{2}$, for the heavy holes. The relative contribution of band $i$ to the total probability is given by $p_{i}$ $=\int_{V} P_{i} d V$, where $i$ is $h h, l h$, or so. Only the $s$ shell $\left(l_{e}=0\right)$ electron states, denoted by $n s^{\sigma}$, are computed and analyzed.

Different boundary conditions for even and odd states produce different secular equations of the single-band Hamiltonian for the conduction band. Not only the number of zeros in the $z$ direction matters for the ordering of the states, but the spatial localization in the plane of the quantum dot layers brings about nontrivial ordering patterns of the electron states. For example, a single zero exists in the wave functions of the $2 s^{+}$and $1 s^{-}$states, positioned along the $\rho$ and $z$ directions, respectively. Therefore, depending on the dimensions of the quantum dot, the $2 s^{+}$energy level may in principle lie below $1 s^{-}$level or vice versa. The classification of the valence band states by the number of zeros is not easily manageable. However, the crossings between states of the same parity and the same $f$ are forbidden. ${ }^{23}$

The envelope functions in both the valence and conduction bands are expanded into

$$
\chi_{\ln m}^{ \pm}(\varphi, \rho, z)=\frac{1}{\sqrt{2 \pi}} \exp (i l \varphi) g_{n(l)}(\rho) f_{m}^{ \pm}(z) .
$$

The $\rho$-dependent part of the basis function is given by

$$
g_{n(l)}(\rho)=\frac{\sqrt{2}}{R_{t}} \frac{1}{\left|J_{|l|+1}\left(k_{n(l)} R_{t}\right)\right|} J_{l}\left(k_{n(l)} \rho\right) .
$$

The $z$-dependent part of the basis function consists of the standing waves of a well-defined parity:

$$
\begin{aligned}
& f_{m}^{+}(z)=\frac{1}{\sqrt{H_{z}}} \cos \left(\frac{m \pi}{2 H_{z}} z\right), \quad m=1,3,5, \ldots, \\
& f_{m}^{-}(z)=\frac{1}{\sqrt{H_{z}}} \sin \left(\frac{m \pi}{2 H_{z}} z\right), \quad m=2,4,6 \ldots
\end{aligned}
$$

In Eqs. (20), (21a), and (21b), $R_{t}$ and $H_{z}$ denote the radius and the half-height of the expansion cylinder, $k_{n(l)} R_{t}$ is the $n$th zero of the Bessel function of order $l$, and $m$ the order of the $z$-dependent $\cos / \mathrm{sin}$ basis function. $l$ is computed for the given $f$ and $j$. Matrix elements between $\chi$ functions due to all operators in Eq. (12) are given in the Appendix. We assumed a step variation of the material parameters; thus, all matrix elements are composed of products of six different types of matrix elements between $g_{n(l)}$ functions, shown in Table II, 
TABLE II. Matrix elements between $g$ functions used in the calculation of the Hamiltonian matrix.

\begin{tabular}{lc}
\hline \hline Type & Expression \\
\hline$\mu_{\rho, 1}$ & $\left\langle g_{n^{\prime}\left(l^{\prime}\right)}\left|\frac{\partial}{\partial \rho}\right| g_{n(l)}\right\rangle$ \\
$\mu_{\rho, 2}$ & $\left\langle g_{n^{\prime}\left(l^{\prime}\right)}\left|\frac{1}{\rho}\right| g_{n(l)}\right\rangle$ \\
$\mu_{\rho, 3}$ & $\left\langle g_{n^{\prime}\left(l^{\prime}\right)}\left|\frac{\partial^{2}}{\partial \rho^{2}}\right| g_{n(l)}\right\rangle$ \\
$\mu_{\rho, 4}$ & $\left\langle g_{n^{\prime}\left(l^{\prime}\right)}\left|\frac{1}{\rho} \frac{\partial}{\partial \rho}\right| g_{n(l)}\right\rangle$ \\
$\mu_{\rho, 5}$ & $\left\langle g_{n^{\prime}\left(l^{\prime}\right)}\left|\frac{1}{\rho^{2}}\right| g_{n(l)}\right\rangle$ \\
$\mu_{\rho, 6}$ & $\left\langle g_{n^{\prime}\left(l^{\prime}\right)} \mid g_{n(l)}\right\rangle$ \\
\hline \hline
\end{tabular}

and three different types of matrix elements between $\sin / \cos$ functions, displayed in Table III, and are also supplemented by the boundary terms, which are explicitly given in the Appendix. Due to the Kramers degeneracy and the Hermiticity of the multiband Hamiltonian, out of 36 only 13 blocks of the different matrix elements should be stored in the computer memory. A peculiar characteristic of the zone center basis states of Eqs. (15a)-(15f) is that real symmetric Hamiltonian matrices are formed. Those are diagonalized by the appropriate routine from the ARPACK package. ${ }^{30}+f$ and $-f$ states exhibit Kramers degeneracy; therefore, states of a single — say, positive - sign may only be considered.

In our Hartree-type exciton state calculation we assume that the Coulomb interaction between the electrons and the holes depends only on the probability densities of the ground states in the conduction and the valence bands. The modified effective-mass equations for the electrons and the holes are given by

$$
\left(H_{c}-\frac{q^{2}}{4 \pi \epsilon_{0} \epsilon_{R}} \int_{\Omega_{h}} \frac{\sum_{i=1}^{6}\left|F_{i 1}\right|^{2}}{\left|\mathbf{r}_{e}-\mathbf{r}_{h}\right|} d \Omega_{h}\right) \Psi_{c}^{e h}=E_{c}^{e h} \Psi_{c}^{e h}
$$

and

$$
\left(H_{v}+I \frac{q^{2}}{4 \pi \epsilon_{0} \epsilon_{R}} \int_{\Omega_{e}} \frac{\left|\Psi_{c 1}\right|^{2}}{\left|\mathbf{r}_{e}-\mathbf{r}_{h}\right|} d \Omega_{e}\right) F^{e h}=E_{v}^{e h} F^{e h}
$$

respectively. Here $F_{i 1}$ and $\Psi_{c 1}$ denote the envelope functions of the ground states in the valence and conduction bands, respectively, the superscript $e h$ indicates that the Coulomb interaction between electrons and holes is taken into account, $\Omega_{h}$ and $\Omega_{e}$ denote the spaces defined by the hole and the electron coordinates, respectively, $q$ is the electron charge, $\epsilon_{R}$ is the relative permittivity, and $I$ is the unity matrix. Equation (22) and (23) are solved iteratively until the prescribed tolerance for $E_{c 1}^{e h}-E_{v 1}^{e h}$ is achieved. The expansion into the basis, Eq. (19), may suggest the application of
TABLE III. Three different types of matrix elements between $f_{m}$ functions employed in the calculation of the Hamiltonian matrix.

\begin{tabular}{lc}
\hline \hline Type & Expression \\
\hline$\mu_{z, 0}$ & $\left\langle f_{m^{\prime}} \mid f_{m}\right\rangle$ \\
$\mu_{z, 1}$ & $\left\langle f_{m^{\prime}}\left|\frac{d}{d z}\right| f_{m}\right\rangle$ \\
& $\left\langle f_{m^{\prime}}\left|\frac{d^{2}}{d z^{2}}\right| f_{m}\right\rangle$ \\
$\mu_{z, 2}$ & \\
\hline \hline
\end{tabular}

the Fourier transform, ${ }^{23}$ but we found it to be convenient to complete the calculation in real space. ${ }^{15}$ When the iteration is completed, the exciton transition energy is computed as

$$
\begin{aligned}
E_{e x c}= & E_{c 1}^{e h}-E_{v 1}^{e h}+\frac{q^{2}}{4 \pi \epsilon_{0} \epsilon_{R}} \\
& \times \int_{\Omega_{h}} d \Omega_{h} \int_{\Omega_{e}} d \Omega_{e} \frac{\left|\Psi_{c 1}\right|^{2} \sum_{i=1}^{6}\left|F_{i 1}\right|^{2}}{\left|\mathbf{r}_{e}-\mathbf{r}_{h}\right|} .
\end{aligned}
$$

We determine the binding energy as

$$
E_{b i n}=\left(E_{c 1}-E_{v 1}\right)-E_{e x c}
$$

where $\left(E_{c 1}-E_{v 1}\right)$ denote the difference between the singleelectron and -hole energies uncorrelated by the Coulomb interaction.

\section{NUMERICAL RESULTS AND DISCUSSION}

For the calculation of the electronic structure, all parameters, except the electron effective mass in InGaP, are taken from Ref. 11 and are given in Table I. For the electron effective mass in the InGaP matrix, the more recent value of $m_{e}$ $=0.125 m_{0}$ is used. ${ }^{31}$ The experimental value of the relative permittivity $\epsilon_{R}$ in InP is adopted for both InP and InGaP. Both the radius and half-height of the expansion cylinder (i.e., the simulation region) were assumed to be $32 \mathrm{~nm}$. Ten Bessel and 50 sin or cos functions are employed in the calculation. Our expansion parameters are very similar to those for the conical quantum dots used in Ref. 32. The Hartree procedure is iterated until the difference of the ground-state energies $E_{c 1}^{e h}-E_{v 1}^{e h}$ in the two subsequent iterations deviate by less than $0.1 \mathrm{meV}$, which is achieved typically in 3 iterations.

The effective potentials for the electrons and heavy and light holes along the $z$ and $\rho$ directions are displayed in Figs. 4(a)-4(c) and 4(d)-4(f), respectively, for a quantum dot with radius $R=8 \mathrm{~nm}$ and height $h=2.5 \mathrm{~nm}$. Because the IE strain distribution is uniformly sampled with the step size $0.25 \mathrm{~nm}$, the effective potentials at the $z= \pm h$ interfaces are not completely abrupt, while in the CM calculation a more refined mesh is applied near the boundary. We consider this slight difference between the two strain distributions unimportant for the conclusions derived in this section. When 


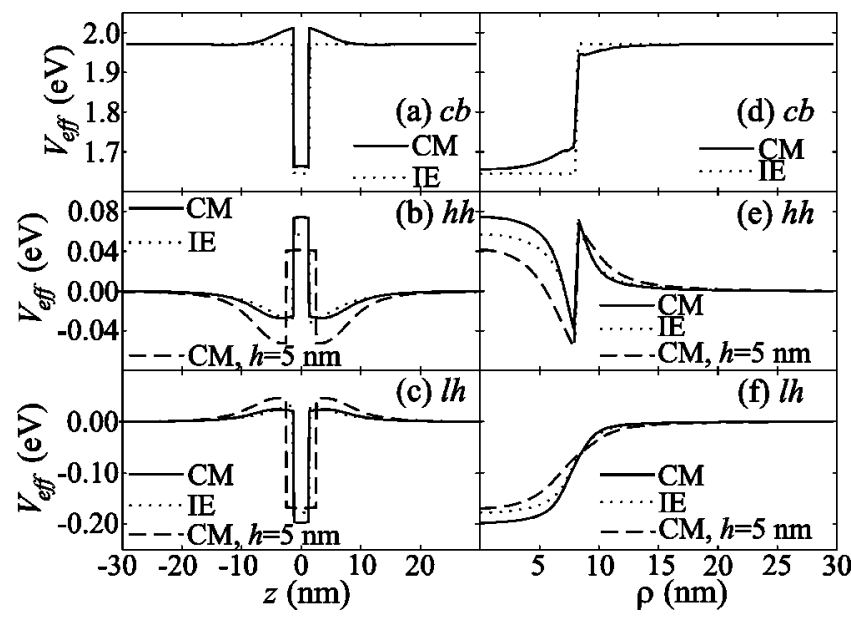

FIG. 4. (The left panel.) The effective potentials for the electron and heavy and light holes along the $z$ axis in a 2.5-nm-high quantum dot with radius $8 \mathrm{~nm}$ for the two different strain distributions. (a) The bottom of the conduction band $(c b)$. (b) The heavy-hole $(h h)$ effective potential. (c) The light-hole $(l h)$ effective potential (right panel). The effective potentials for the electrons, heavy and light holes along the radial axis for $z=0$ in a 2.5-nm-high quantum dot with radius $8 \mathrm{~nm}$ for the two different strain distributions. (d) The bottom of the conduction band $(c b)$. (e) The effective potential for the heavy holes $(h h)$. (f) The effective potential for the light holes $(l h)$. The solid lines in all figures denote the CM calculated effective potentials and the dotted lines the effective potential obtained by the IE theory, while the dashed lines in (b), (c), (e), and (f) represent the effective potentials for a $h=5 \mathrm{~nm}$ high quantum dot.

compared with the CM model, the IE theory leads to a deeper effective potential well for the electrons and a shallower one for the heavy holes. As a matter of fact, the CMcalculated value of the conduction band potential in the center of the dot with respect to the bottom of the conduction band in the matrix amounts to $306.3 \mathrm{meV}$, whereas the same quantity calculated from the IE theory is equal to $324.5 \mathrm{meV}$. For the heavy holes, the CM computed effective potential in the center of the dot equals $80.3 \mathrm{meV}$, while $56.9 \mathrm{meV}$ is found from the IE theory. The vanishing hydrostatic strain in the IE calculation provides the minimum of the elastic energy, but it gives an incorrect effective potential in the barrier, as shown in Figs. 4(a) and 4(d). Notice that the effective potential wells for the electrons and the heavy holes favor confinement inside the dot, while the light holes are expelled from the dot to regions in InGaP near the dot boundary. The barrier for the light holes in the dot is higher within the CM model as compared to the result from the IE calculation. As already explained, $\varepsilon_{a v}$ and $\left\langle\varepsilon_{a v}\right\rangle$ depicted in Figs. 3(a) and 3 (c) have different shapes, which accounts for the difference between the effective potentials for the heavy and the light holes calculated by the two strain models outside the dot, as shown in Figs. 4(b) and 4(c). It seems that the effective potential for the conduction band shown in Fig. 4(a) is not affected by the peculiar distribution of $\left\langle\varepsilon_{a v}\right\rangle$. Since the hydrostatic part in the effective potentials for the holes is much smaller (cf. $a_{c} \sim-7 \mathrm{eV}$ with $a_{v}=0.4 \mathrm{eV}$; see Table I), the "wavy" shapes of the effective potentials for the heavy and light holes occur due to the tetragonal strain. ${ }^{1}$ In the IE cal- culation of the effective potentials for the holes, the slight variations near the dot boundary are smeared out. But both strain calculations gives electrons and heavy holes located inside the dot, while the light holes are confined in the surrounding InGaP matrix near the $z= \pm h / 2$ interfaces. For the $\rho$ direction, the effective potentials for the electrons, heavy and light holes differ substantially, as shown in Figs. 4(d)4(f). The discontinuity of the effective potential for the heavy holes shown in Fig. 4(e) indicates that some small part of the heavy-hole envelope function can be found in the narrow quantum well located in InGaP near the boundary, but our calculation indicates that this part is rather negligible, at least in the ground hole states with $S_{3 / 2}^{+}$and $S_{1 / 2}^{+}$symmetries. The effective potential for the light holes shown in Fig. 4(f) consists of the relatively flat region near the center and the continuous decay in the vicinity of the dot boundary. Figures 4(b), 4(c), 4(e), and 4(f) also show the effective potential wells for the heavy and the light holes in the $h=5 \mathrm{~nm}$ thick quantum disk. When compared with the $h=2.5 \mathrm{~nm}$ case, the effective potential well for the heavy holes is shallower and the effective potential wells for the light holes deeper. Furthermore, the height of the barrier for the heavy holes in the matrix increases, while for the light holes in the dot decreases. These changes have a profound influence on the electronic structure, as illustrated below.

We are interested in the energies near the top of the valence band, where the split-off part in the mixed hole state is negligible, and the heavy-hole and the light-hole zone center states govern the electronic structure. It turns out that the ground state has $f=3 / 2$. For this value of $f$, the heavy holes have $l=0$ and $l=-3$ and the light holes $l=-1$ and $l=$ -2 . The main parts in the probability density belong to the envelope functions with the smallest absolute values of envelope quantum numbers, $|l|=0$ and $|l|=1$ in the $f=3 / 2$ case, and those envelope functions will only be discussed below. Due to the smaller $|l|$ (smaller kinetic energy) and deeper effective potential well for the heavy holes, the $1 S_{3 / 2}^{+}$ state is dominated by the heavy-hole part, as shown in the left part of Fig. 5(a). The light-hole part is displayed in the right part of Fig. 5(a). The separation between the consecutive contour lines in the right part is 100 times smaller than the separation in the left part, indicating a negligible contribution of the light-hole part in this state. The heavy-hole part amounts to $p_{h h}=0.855$, while the light-hole part is $p_{l h}$ $=0.129$. Since the effective potential wells for the light holes created by the inhomogeneous strain distribution are located on the top and the bottom of the quantum disk, the light-hole part is located below and above the disk, but $p_{l h}<p_{h h}$ due to the larger envelope angular momentum of the light holes. The location of the light-hole states is similar to the location of the B states in the notation of Pryor et al. ${ }^{11}$ for truncated pyramidal quantum dots. As a consequence of the different envelope angular momenta, shapes of the probability densities are quite different for the two bands. The light-hole cloud is positioned at $\rho \neq 0$, while the peak of the heavy-hole cloud is in the center of the dot.

For the $1 S_{1 / 2}^{+}$state, the envelope angular quantum number of the lowest magnitude $l=0$ belongs to the light hole; thus, 

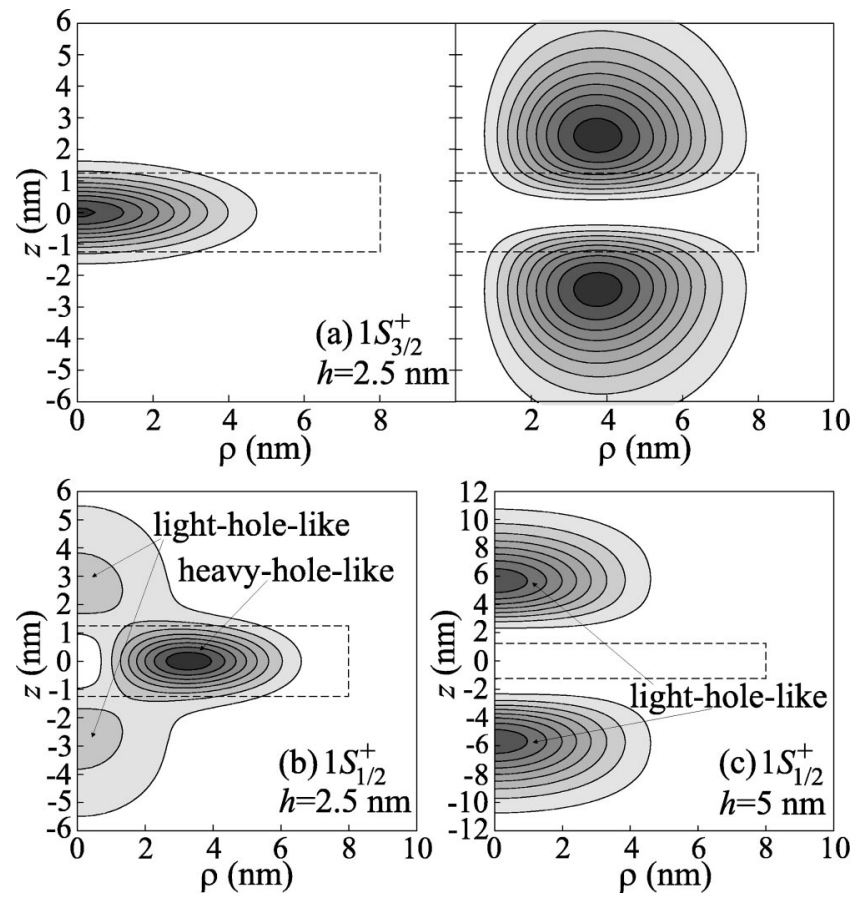

FIG. 5. The probability densities of the $1 S_{3 / 2}^{+}$and $1 S_{1 / 2}^{+}$states as calculated from the CM determined strain distributions in a quantum dot with radius $8 \mathrm{~nm}$. Darker regions denote higher probability density. (a) The probability density of the $1 S_{3 / 2}^{+}$state for a $h$ $=2.5 \mathrm{~nm}$ high quantum dot. The light-hole part in this state is displayed in the right panel. Note that the separation between the two consecutive contour lines in the main figure equals $1.5 \times 10^{-3}$ $\mathrm{nm}^{-3 / 2}$, while in the right panel it corresponds to a 100 times smaller interval. (b) The $1 S_{1 / 2}^{+}$probability density in the $h$ $=2.5 \mathrm{~nm}$ high quantum dot. The heavy-hole and the light-hole parts are explicitly indicated in the figure. (c) The probability density of the $1 S_{1 / 2}^{+}$state in a $h=5 \mathrm{~nm}$ high quantum dot. For $5 \mathrm{~nm}$ high quantum dots, the ground hole state has a $S_{1 / 2}^{+}$symmetry.

two peaks of the light-hole probability density occur, as indicated in Fig. 5(b). On the other hand, the peak of the ringshaped heavy-hole cloud $(l=-1$ and $l=2)$ is located inside the dot, but shifted towards the $\rho=R$ boundary. The heavyhole envelope orbital momentum is larger in this state than in the $1 S_{3 / 2}^{+}$state, and consequently the heavy-hole part is smaller in the $1 S_{1 / 2}^{+}$state $\left(p_{h h}=0.718\right.$ and $\left.p_{l h}=0.246\right)$. A noticeable effect is the increase of the width of the heavyhole cloud near $\rho=0$. This effect is due to the finite penetration depth of the light-hole cloud in the dot region, which effectively increases the width of the probability density in the $z$ direction. Due to the shallow effective potential wells for the light holes [see Figs. 4(b), 4(c), 4(e), and 4(f)] the heavy-hole part dominates also in the $1 S_{1 / 2}^{+}$state. The probability densities of the $1 S_{3 / 2}^{+}$and $1 S_{1 / 2}^{+}$states for the IE calculated strain distributions are similar to the diagrams depicted in Figs. 5(a) and 5(b). Due to the simultaneous deepening of the effective potential well for the heavy holes and shallowing of the effective potential wells for the light holes, as explained above, the ground state in the $h=5 \mathrm{~nm}$ thick quantum dot has $S_{1 / 2}^{+}$symmetry. The probability density consists of two light-hole clouds, positioned above and be-

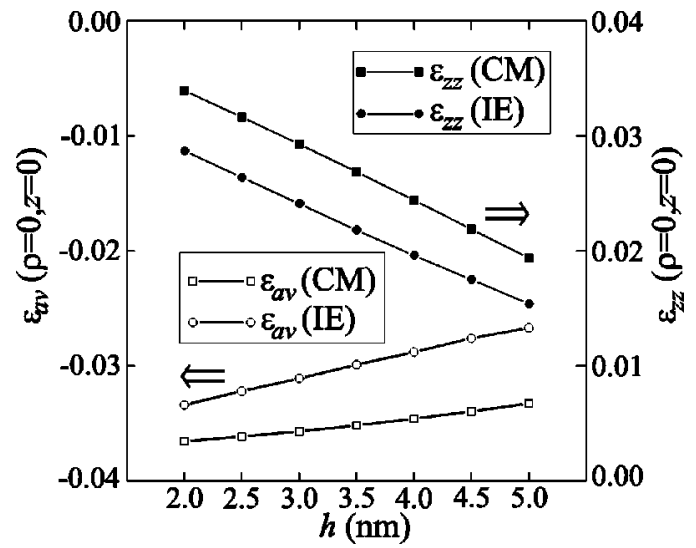

FIG. 6. The dependence of the average of the in-plane strain components $\varepsilon_{a v}=\left(\varepsilon_{x x}+\varepsilon_{y y}\right) / 2$ and $\varepsilon_{z z}$ in the center of the quantum dot as a function of the dot height.

low the quantum disk, as shown in Fig. 5(c). In this quantum dot the effective potential wells for the light holes are deeper ( $\sim 46 \mathrm{meV}$ near the $z= \pm h$ boundaries) than the effective potential well for the heavy holes $(\sim 41 \mathrm{meV}$ in the center of the dot). Furthermore, due to the increased width of the effective potential wells for the light holes, the heavy-hole contribution to the probability density in the $1 S_{1 / 2}^{+}$state is negligible for $h=5 \mathrm{~nm}$. We found that $p_{l h}=0.803$ and $p_{h h}$ $=0.102$ in the ground state for $h=5 \mathrm{~nm}$ high quantum dot.

The electronic structure for the strain distributions calculated for the two models are compared for a set of quantum dots, having the same radius $R=8 \mathrm{~nm}$ and heights in the range $2-5 \mathrm{~nm}$. In the multiband effective-mass calculation applied to self-assembled quantum dots, all dimensions are usually mutually scaled. ${ }^{7}$ Since the strain distribution is virtually independent of this scaling, ${ }^{16}$ the electronic structure modification is brought about only by the size variation. If a single dimension is scaled, however, the strain fields change, influencing the electronic structure in a nontrivial way. The values of $\varepsilon_{a v}$ and $\varepsilon_{z z}$ in the center of the quantum dot are shown in Fig. 6. One may notice that $\varepsilon_{z z}$ decreases much faster than $\varepsilon_{a v}$ increases when the height of the quantum dot increases and also that the magnitudes of all diagonal strain components are larger if calculated by the CM method.

The energies of the two lowest $s$ shell states are displayed in Fig. 7(a). The energies of the $S_{1 / 2}^{+}$and $S_{3 / 2}^{+}$ground states are shown in Fig. 7(b), the energies of the higher hole states of even and odd parity calculated by the CM model are displayed in Figs. 7(c) and 7(d), respectively, and the comparison of the exciton energies computed for the three strain calculations is shown in Fig. 7(e). We found that the anisotropic strain distributions produce the same features in the electronic structure as the strain distributions calculated from the IE theory. The similarities and differences between the two calculations are summarized below.

First, as a consequence of the zero hydrostatic strain in InGaP and the smaller effective potential for the electrons shown in Fig. 4(a), the ground electron energy is underestimated when the IE calculated strain distributions are used. Energies of the $1 S_{3 / 2}^{+}$and $1 S_{1 / 2}^{+}$states are also underestimated by the IE theory, since the main part in the probability den- 


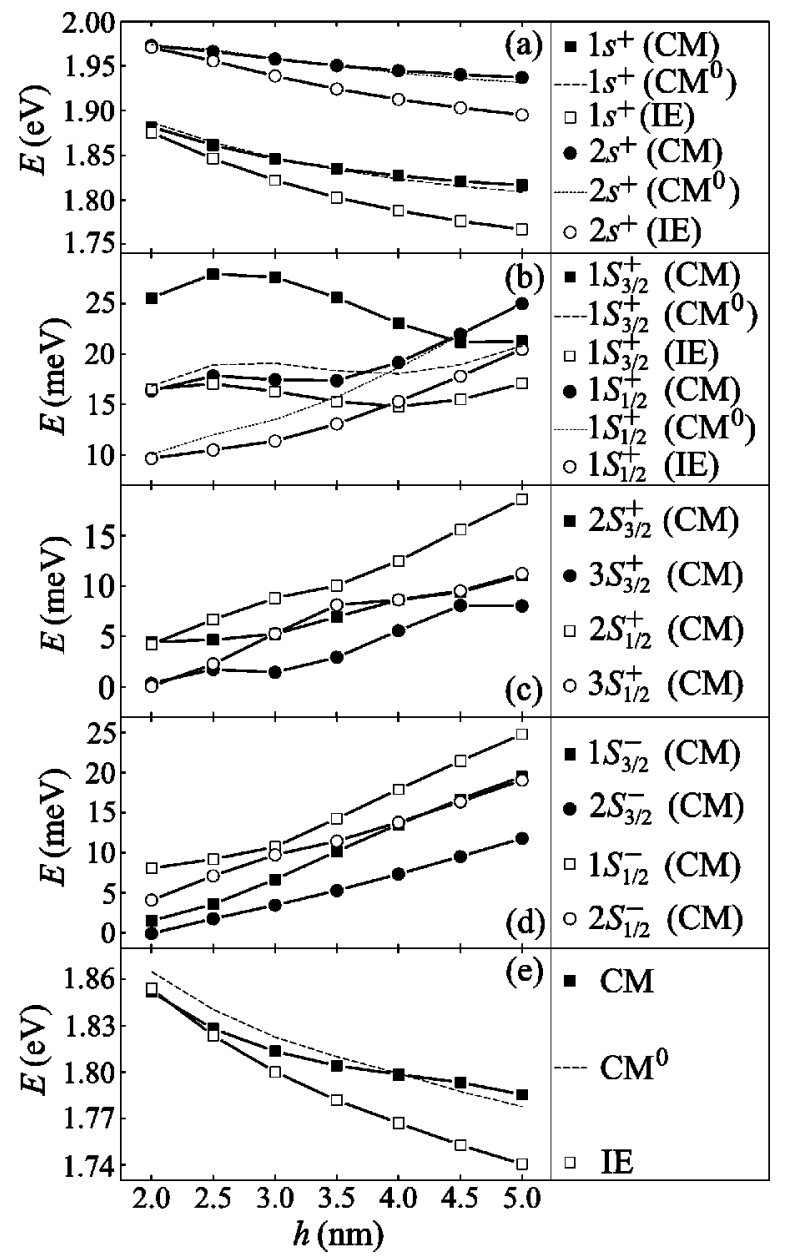

FIG. 7. The electron and the hole energies calculated for the strain distributions determined by the three types of the strain calculation. The quantum dot height varies in the range $2-5 \mathrm{~nm}$, while the radius is fixed at $R=8 \mathrm{~nm}$. (a) The energies of the two lowest $s$ shell electron states. (b) The dependence of the energies of the $1 S_{1 / 2}^{+}$ and $1 S_{3 / 2}^{+}$hole states on the height of the quantum dot. Note the reversal of the angular momentum of the hole ground state from $3 \hbar / 2$ to $\hbar / 2$. (c) Higher hole energy levels of $S_{3 / 2}^{+}$and $S_{1 / 2}^{+}$symmetries for the strain distributions calculated by the CM method. (d) The first two hole levels of $S_{3 / 2}^{-}$and $S_{1 / 2}^{-}$symmetry, as they vary with the height of the quantum dot. In both (c) and (d), there exist anticrossings between subsequent hole levels of the same symmetry, but the two states of different parity may cross. (e) The dependence of the exciton energy on the height of the quantum dot for a fixed dot radius $R=8 \mathrm{~nm}$. The result of the $\mathrm{CM}^{0}$ calculation, shown by the dashed line, does not deviate much from the CM calculation.

sity belongs to the heavy holes, and the effective potential for the heavy holes is lower if calculated by the IE theory.

Second, due to the smaller effective potential barrier for the electrons in InGaP and larger increase of $\varepsilon_{\text {hyd }}$ calculated by the IE theory, the IE-calculated separation between the two lowest $s$ shell states becomes larger than the separation between the CM-calculated energy levels when the height of the quantum dot increases.

Third, the energies of the ground electron state and the exciton energy decrease monotonically with the height of the quantum dot. On the contrary, the hole ground states for both the IE and the CM strain distributions exhibit peaks at $d_{0}$ $\sim 2.5 \mathrm{~nm}$. For $d<d_{0}$, the influence of the decrease of the effective potential is smaller, and the hole ground state energy increases in response to the increase of the SAQD's height. The situation reverses for $d>d_{0}$, where the hole ground-state energy decreases as a result of the decrease of the confining potential for the heavy holes.

Fourth, due to the different confining potentials for the heavy holes and the light holes, the $z$ component of the total orbital momentum of the ground hole state amounts to $F_{z}$ $=3 \hbar / 2$ for $d<4.5 \mathrm{~nm}$, while it is $F_{z}=\hbar / 2$ for thicker quantum dots. This crossover occurs below $4 \mathrm{~nm}$ if the IE theory is used for the calculation of the strain distribution. The $1 P_{5 / 2}^{+}$state [not shown in Fig. 7(b)] is nearly parallel to the $1 S_{3 / 2}^{+}$state and displaced by approximately $10 \mathrm{meV}$ towards lower energies. It crosses the $1 S_{1 / 2}^{+}$state at approximately 2.5 $\mathrm{nm}$; thus, the two lowest hole states in $h=2 \mathrm{~nm}$ high quantum dot are $1 S_{3 / 2}^{+}$and $1 P_{5 / 2}^{+}$. The deviations from axial symmetry might be assessed by the diagonalization of the full multiband Hamiltonian, Eq. (12), and the three-dimensional Schrödinger equation, given by Eq. (10), but with $\left\langle\varepsilon_{\text {hyd }}\right\rangle$ replaced by $\varepsilon_{\text {hyd }}$ in the CM approach. Since $1 S_{3 / 2}^{+}, 1 S_{1 / 2}^{+}$, and $1 P_{5 / 2}^{+}$are the lowest electron energies in the axially symmetric description for the 2-3-nm-thick quantum dots, the weights of those states may a priori be largest in the full three-dimensional description of the hole spectra.

Fifth, $\mathrm{CM}^{0}$ and $\mathrm{CM}$ calculated electron energies exhibit a very good agreement for the whole explored range of quantum dot heights, as indicated in Fig. 7(a) by the dashed lines. The hole energies determined by the $\mathrm{CM}^{0}$ calculation, however, deviate from the CM result in a more complex way, as displayed in Fig. 7(b) for the ground $1 S_{3 / 2}^{+}$(long dashed line) and $1 S_{1 / 2}^{+}$(short dashed line) states. For thin quantum dots the $1 S_{3 / 2}^{+}$and $1 S_{1 / 2}^{+}$states calculated for the same elastic constants in the dot and barrier are close to the energies extracted from the IE calculation, but when $h$ increases, they approach the CM calculated hole levels and almost coincide with those levels for 5-nm-high quantum dots. Such behavior indicates that the proper description of the hole spectra in cylindrical quantum dots should encompass both anisotropy and the spatial variation of the elastic constants. The crossover between $1 S_{3 / 2}^{+}$and $1 S_{1 / 2}^{+}$states found by the CM and IE calculation is also reproduced for the elastically anisotropic structure consisting of the semiconductors with the same elastic constants. Its position, indicated by the crossing between the dashed lines in Fig. 7(b), almost coincides with the crossover found for the strains computed by the IE theory; i.e., it is shifted towards smaller heights in respect to the CM result.

Sixth, higher hole states show anticrossings, as shown in Figs. 7(c) and 7(d) for the even- and odd-parity states, respectively. Only the CM calculated curves are displayed, but the same qualitative behavior was found for both the IE and $\mathrm{CM}^{0}$ calculations. In addition to the crossings of the states of the different angular momenta, there also exist crossings between the odd and even parity states, which may be demonstrated by comparing Fig. 7(c) with Fig. 7(d). For example, 
we found that the $1 S_{3 / 2}^{+}$state crosses the $2 S_{3 / 2}^{-}$state at approximately $2.5 \mathrm{~nm}$.

Seventh, due to the increase of the localization in the wide quantum wells for the light holes on the top and the bottom, $S_{1 / 2}^{+}$states in the $h=5 \mathrm{~nm}$ high quantum dot appear as doublets. In other words, the system behaves as two coupled quantum dots for the light holes. Since the separation between those "strain-induced" quantum dots is rather large, the levels are nearly degenerate. The envelope functions differ by the parity, but their probability densities almost coincide.

Eighth, the exciton transition energy calculated by the two strain models decreases with increasing $h$. For 2-nm-high quantum dots, the IE computed exciton energy is larger, but decreases faster with an increase of the quantum dot height than the exciton energy calculated by means of the CM method. The exciton binding energy for the CM strain distributions varies between 4.8 and $6.0 \mathrm{meV}$ for $h$ between 2 and $5 \mathrm{~nm}$, and its influence on the obtained trends is smaller than the behavior of the ground electron and hole energies. In other words, the difference between the slopes of the CM and IE calculated curves shown in Fig. 7(e) mainly arises from the different slopes of the CM and IE curves for $E_{1 s^{+}}(h)$ in Fig. 7(a). A slight increase of the slope of the exciton transition energy versus the quantum dot height for $h>4.5 \mathrm{~nm}$ is provided by the crossover of $1 S_{3 / 2}^{+}$and $1 S_{1 / 2}^{+}$ and the increase of the energy of the $1 S_{1 / 2}^{+}$state. Due to the higher electron energies for $h<3 \mathrm{~nm}$ and lower hole energies in the whole range of $h$, the $\mathrm{CM}^{0}$ calculated exciton transition energy is higher than the CM determined one for $h \leqslant 4 \mathrm{~nm}$. However, reversal of the sign of electron energies and a vanishing difference between the ground hole states obtained by the two calculations, reverses the sign of the difference between the $\mathrm{CM}$ and $\mathrm{CM}^{0}$ calculated exciton transition energies for $h>4 \mathrm{~nm}$.

The experimental photoluminescence line located ${ }^{2}$ at $1.800 \mathrm{eV}$ agrees with our result for the 4-nm-thick quantum dots, while for the $h=3 \mathrm{~nm}$ high quantum $\operatorname{dot}^{2}$ we found $1.814 \mathrm{eV}$. For a $h=2 \mathrm{~nm}$ thick quantum dot, the center of mass of the photoluminescence line is $40 \mathrm{meV}$ lower than the theoretical result. ${ }^{3}$ Our calculated energies are higher than those found experimentally which may be due to either the limited applicability of the continuum mechanical model for thin cylindrical quantum dots or experimental uncertainties in the determination of the dot height. In InAs/GaAs quantum dots it was found that the VFF model produces deeper confining potentials for both the electrons and the holes, thereby offering a few tens of meV smaller free electron-hole transition energies than the $\mathrm{CM}$ model. ${ }^{7}$ This may provide the explanation for the difference between the experiment and the theory found here, but the comparison with a VFFtype calculation is out of the scope of the present paper. Moreover, as recently noticed, the choice of the Luttinger parameters and the electron effective mass might be crucial for the accuracy of the electronic structure calculation by the multiband effective-mass theory, ${ }^{33}$ which delivers $\sim 100 \mathrm{meV}$ higher transition energies in InAs/GaAs quantum dots than found by the pseudopotential theory. ${ }^{34}$ There-

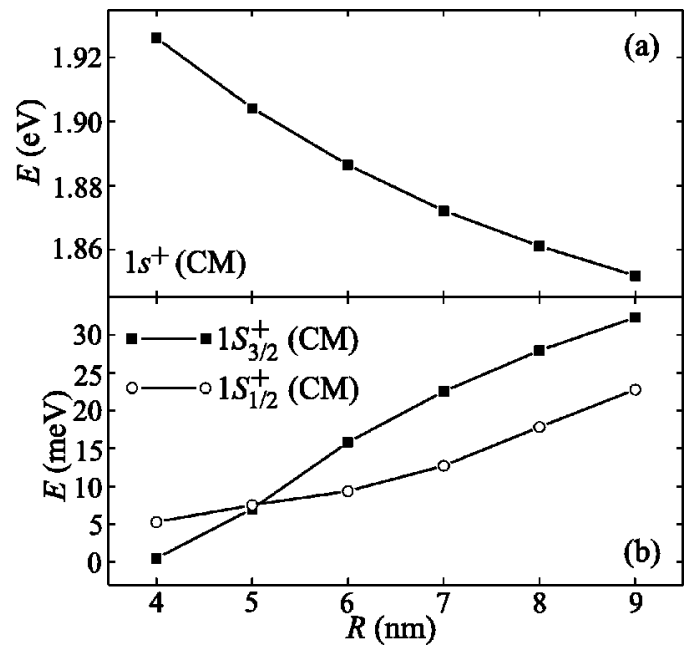

FIG. 8. The dependence of the electron (a) and hole (b) levels on the radius in a $h=2.5 \mathrm{~nm}$ high quantum dot. Due to $R>h$, the decrease of the electron energy is rather small, while the ground hole energies exhibit a crossing at $R \approx 5.1 \mathrm{~nm}$.

fore, our theoretical results provide rather trends and order of magnitude estimates of the electron and hole energies.

Finally, the dependence of the electron and the hole energies on the radius of the quantum dot for a fixed height $h$ $=2.5 \mathrm{~nm}$ is explored. Only the electron and hole energies without Coulomb interaction are calculated. These are shown in Figs. 8(a) and 8(b), respectively. Due to $R>h$, the $1 s^{+}$ state in the conduction band exhibits a weaker dependence on the quantum dot radius than on the quantum dot height [compare Fig. 8(a) with Fig. 7(a)]. The two curves depicting $1 S_{3 / 2}^{+}$and $1 S_{1 / 2}^{+}$states have a different curvature. As for the case of increasing height, the variation of the strain field results into a crossing of the $1 S_{3 / 2}^{+}$and $1 S_{1 / 2}^{+}$states when the radius of the dot decreases.

\section{SUMMARY}

In conclusion, the multiband effective-mass approach for disk-shaped $\mathrm{InP} / \mathrm{InGaP}$ self-assembled quantum dots was presented in this paper. Based on the small in-plane warping term of the kinetic Hamiltonian, the axial approximation was adopted for the kinetic part of the Hamiltonian. We found that the average of the in-plane strain tensor components $\left(\varepsilon_{x x}+\varepsilon_{y y}\right) / 2$ and $\varepsilon_{z z}$ exhibits negligible deviations from axial symmetry, whereas the shear strains are negligible everywhere except near the boundary. Based on these findings, the strain-dependent Hamiltonian was replaced by an axially symmetric one. The electron and hole energy levels were calculated for isotropic and anisotropic strain distributions and compared for a range of quantum dot heights. We found similar qualitative behavior for the two strain calculations, with both the electron and hole energies slightly lower for the IE calculated strain distribution. A crossover of the $f$ $=3 / 2$ to a $f=1 / 2$ hole ground state was found for both strain models. The obtained exciton energies are in reasonable agreement with the experimental results. 


\section{ACKNOWLEDGMENTS}

This work was partially supported by the Flemish Science Foundation (FWO-Vl), the Belgian Interuniversity Attraction Poles (IUAP), the University of Antwerp (GOA and VIS), and the European Commission GROWTH program NANOMAT project, Contract No. G5RD-CT-2001-00545. M.T. was supported by the "Belgian Office for Scientific, Technical and Cultural Cooperation" (BELSPO). K.L.J. was supported by the "Instituut voor de aanmoediging van Innovatie door Wetenschap en Technologie in Vlaanderen" (IWTVl). We acknowledge fruitful discussions with Professor J. H. Davies, Dr. Z. Ikonić, Dr. B. Partoens, M. Korkusinski, Dr. O. Stier, Dr. M. Hayne, Professor M. Skolnick, Dr. P. Hawrylak, and Professor V. Moshchalkov.

\section{APPENDIX}

Luttinger parameters and their linear combinations in the multiband effective-mass Hamiltonian are constant in both the dot and the matrix, but vary abruptly at the dot boundary. Matrix elements between different $\chi$ functions, Eq. (19), consist of boundary terms originating from the derivatives of $\eta$ and those where $\eta$ is the scaling function. The latter are calculated as

$$
M_{\eta, i, j}=\eta_{m} \mu_{\rho m, i} \mu_{z m, j}+\left(\eta_{d}-\eta_{m}\right) \mu_{\rho d, i} \mu_{z d, j},
$$

where the first letter in the subscript of the one-dimensional matrix elements $\mu$ on the right-hand side denotes the direction ( $\rho$ or $z$ ), the second subscript refers to the domain of the integration ( $m$ is for the whole expansion cylinder and $d$ for the dot), and the third letter is the label of the type of the matrix element. Six different types of matrix elements in the $\rho$ direction, and three types for the $z$ direction are shown in Tables II and III, respectively.

Furthermore, it may be shown that all boundary terms depend on one of the following functions:

$$
\begin{gathered}
\Delta_{\rho, 0}=-R g_{n^{\prime}\left(l^{\prime}\right)}(R) g_{n(l)}(R), \\
\Delta_{\rho, 1}=-\left.R g_{n^{\prime}\left(l^{\prime}\right)}(R) \frac{d g_{n(l)}}{d \rho}\right|_{\rho=R}, \\
\Delta_{z, 0}=f_{m^{\prime}}(-d) f_{m}(-d)-f_{m^{\prime}}(+d) f_{m}(+d), \\
\Delta_{z, 1}=\left.f_{m^{\prime}}(-d) \frac{d f_{m}}{d z}\right|_{-d}-\left.f_{m^{\prime}}(d) \frac{d f_{m}}{d z}\right|_{+d} .
\end{gathered}
$$

\section{Diagonal terms and $Q_{k}$ matrix element in the multiband effective-mass Hamiltonian}

All diagonal and $Q_{k}$ matrix elements of the kinetic part of the Hamiltonian (12), generally denoted by $D_{k}$, have the form

$$
D_{k}=D_{\rho}+D_{z}=\eta_{\rho}\left(k_{x}^{2}+k_{y}^{2}\right)+\eta_{z} k_{z}^{2} .
$$

$\eta_{r}$ and $\eta_{z}$ are linear combinations of the Luttinger parameters multiplied by $\hbar^{2} /\left(2 m_{0}\right)$. The $\rho$ dependent part of this operator is equal to

$$
D_{\rho}=-\eta_{\rho} \frac{\partial^{2}}{\partial \rho^{2}}-\frac{\eta_{\rho}}{\rho} \frac{\partial}{\partial \rho}-\frac{\eta_{\rho}}{\rho^{2}} \frac{\partial^{2}}{\partial \varphi^{2}}-\frac{\partial \eta_{\rho}}{\partial \rho} \frac{\partial}{\partial \rho} .
$$

The matrix element between the $\chi_{l^{\prime} n^{\prime} m^{\prime}}$ and $\chi_{l n m}$ states is expressed as

$$
\begin{aligned}
M_{D_{\rho}}= & \delta_{l-l^{\prime}}\left[-M_{\eta_{\rho}, 3,0}-M_{\eta_{\rho}, 4,0}+l^{2} M_{\eta_{\rho}, 5,0}\right. \\
& \left.-\left(\eta_{\rho d}-\eta_{\rho m}\right) \Delta_{\rho, 1} \mu_{z d, 0}\right] .
\end{aligned}
$$

The $z$-dependent part of $D_{k}$ is given by

$$
D_{z}=-\frac{\partial \eta_{z}}{\partial z} \frac{\partial}{\partial z}-\eta_{z} \frac{\partial^{2}}{\partial z^{2}}
$$

The matrix element between $\chi_{l^{\prime} n^{\prime} m^{\prime}}$ and $\chi_{\operatorname{lnm}}$ reads

$$
M_{D_{z}}=\delta_{l-l^{\prime}}\left[-M_{\eta_{z}, 6,2}-\left(\eta_{z d}-\eta_{z m}\right) \Delta_{z, 1} \mu_{\rho d, 6}\right] .
$$

\section{2. $S_{k}$ matrix element of the multiband effective-mass Hamiltonian} form

In cylindrical coordinates, the $S_{k}$ term in Eq. (12) has the

$$
\begin{aligned}
s_{k}= & \frac{\hbar^{2}}{2 m_{0}} \frac{\sqrt{6}}{2} e^{-i \varphi}\left(-\frac{\partial \gamma_{3}}{\partial z} \frac{\partial}{\partial \rho}+\frac{i}{\rho} \frac{\partial \gamma_{3}}{\partial z} \frac{\partial}{\partial \varphi}-\frac{\partial \gamma_{3}}{\partial \rho} \frac{\partial}{\partial z}\right. \\
& \left.+\frac{2 i}{\rho} \gamma_{3} \frac{\partial^{2}}{\partial \varphi \partial z}-2 \gamma_{3} \frac{\partial^{2}}{\partial \rho \partial z}\right),
\end{aligned}
$$

and the matrix element is given by

$$
\left\langle l^{\prime} n^{\prime} m^{\prime}\left|S_{k}\right| \ln m\right\rangle=\frac{\hbar^{2}}{2 m_{0}} \frac{\sqrt{6}}{2} \delta_{l-1-l^{\prime}} \sum_{i=1}^{5} M_{S i},
$$

where

$$
\begin{gathered}
M_{S 1}=-\left(\gamma_{3 d}-\gamma_{3 m}\right) \Delta_{z, 0} \mu_{\rho d, 1}, \\
M_{S 2}=-l\left(\gamma_{3 d}-\gamma_{3 m}\right) \Delta_{z, 0} \mu_{\rho d, 2}, \\
M_{S 3}=-\left(\gamma_{3 d}-\gamma_{3 m}\right) \Delta_{\rho, 0} \mu_{z d, 1}, \\
M_{S 4}=-2 M_{\gamma_{3}, 1,1}, \\
M_{S 5}=-2 l M_{\gamma_{3}, 2,1} .
\end{gathered}
$$

\section{3. $\boldsymbol{R}_{\boldsymbol{k}}$ matrix element of the axially symmetric multiband effective-mass Hamiltonian}

The $R_{k}$ matrix element in Eq. (12) in cylindrical coordinates is given by

$$
\begin{aligned}
R_{k}= & \frac{\hbar^{2}}{2 m_{0}} \sqrt{3} e^{-i 2 \varphi}\left(\frac{1}{\rho} \bar{\gamma} \frac{\partial}{\partial \rho}-\frac{\partial \bar{\gamma}}{\partial \rho} \frac{\partial}{\partial \rho}-\bar{\gamma} \frac{2 i}{\rho^{2}} \frac{\partial}{\partial \varphi}+\frac{i}{\rho} \frac{\partial \gamma_{a v}}{\partial \rho} \frac{\partial}{\partial \varphi}\right. \\
& \left.-\bar{\gamma} \frac{\partial^{2}}{\partial \rho^{2}}+\frac{\bar{\gamma}}{\rho^{2}} \frac{\partial^{2}}{\partial \varphi^{2}}+\frac{2 i}{\rho} \bar{\gamma} \frac{\partial^{2}}{\partial \varphi \partial \rho}\right),
\end{aligned}
$$


where $\bar{\gamma}=\left(\gamma_{2}+\gamma_{3}\right) / 2$. Matrix elements due to the $R_{k}$ operator in the multiband Hamiltonian are therefore

$$
\left\langle l^{\prime} n^{\prime} m^{\prime}\left|R_{k}\right| \operatorname{lnm}\right\rangle=\frac{\hbar^{2}}{2 m_{0}} \sqrt{3} \delta_{l-2-l^{\prime}} \sum_{i=1}^{5} M_{R i},
$$

where

$$
M_{R 1}=-\left(\bar{\gamma}_{d}-\bar{\gamma}_{m}\right) \Delta_{\rho, 1} \mu_{z d, 0},
$$

$$
\begin{gathered}
M_{R 2}=-\left(\bar{\gamma}_{d}-\bar{\gamma}_{m}\right) \frac{l}{R} \Delta_{\rho, 0} \mu_{z d, 0}, \\
M_{R 3}=-M_{\bar{\gamma}, 3,0}, \\
M_{R 4}=(1-2 l) M_{\bar{\gamma}, 4,0}, \\
M_{R 5}=l(2-l) M_{\bar{\gamma}, 5,0} .
\end{gathered}
$$

*On leave of absence from: Faculty of Electrical Engineering, University of Belgrade, Yugoslavia. Electronic address: tadic@uia.ua.ac.be

†Electronic address: peeters@uia.ua.ac.be

${ }^{1}$ D. Bimberg, M. Grundmann, and N. N. Ledentsov, Quantum Dot Heterostructures (Wiley, Chichester, 1999).

${ }^{2}$ M.K. Zundel, P. Specht, K. Eberl, N.Y. Jin-Phillipp, and F. Phillipp, Appl. Phys. Lett. 71, 2972 (1997).

${ }^{3}$ M. Hayne, R. Provoost, M.K. Zundel, Y.M. Manz, K. Eberl, and V.V. Moshchalkov, Phys. Rev. B 62, 10324 (2000).

${ }^{4}$ M. Bayer, P. Hawrylak, K. Hinzer, S. Fafard, M. Korkusinski, Z.R. Wasilewski, O. Stern, and A. Forchel, Science 291, 451 (2001).

${ }^{5}$ M. Grundmann, O. Stier, and D. Bimberg, Phys. Rev. B 52, 11969 (1995).

${ }^{6}$ C. Pryor, J. Kim, L.W. Wang, A.J. Williamson, and A. Zunger, J. Appl. Phys. 83, 2548 (1998).

${ }^{7}$ O. Stier, M. Grundmann, and D. Bimberg, Phys. Rev. B 59, 5688 (1999).

${ }^{8}$ Y. Kikuchi, H. Sugii, and K. Shintani, J. Appl. Phys. 89, 1191 (2001).

${ }^{9}$ J.H. Davies, J. Appl. Phys. 84, 1358 (1998).

${ }^{10}$ L.-W. Wang, J. Kim, and A. Zunger, Phys. Rev. B 59, 5678 (1999).

${ }^{11}$ C. Pryor, M.-E. Pistol, and L. Samuelson, Phys. Rev. B 56, 10404 (1997).

${ }^{12}$ M. Korkusiński and P. Hawrylak, Phys. Rev. B 63, 195311 (2001).

${ }^{13}$ A.J. Davies, The Finite Element Method (Clarendon Press, Oxford, 1980).

${ }^{14}$ A. Szabo and N.S. Ostlund, Modern Quantum Chemistry
(McGraw-Hill, New York, 1989).

${ }^{15}$ K.L. Janssens, B. Partoens, and F.M. Peeters, Phys. Rev. B 64, 155324 (2001).

${ }^{16}$ B. Jogai, J. Appl. Phys. 88, 5050 (2000).

${ }^{17}$ L.D. Landau and E.M. Lifshitz, Theory of Elasticity (Butterworth-Heinemann, Oxford, 1995).

${ }^{18}$ J.R. Downes, D.A. Faux, and E.P. O’Reilly, J. Appl. Phys. 81, 6700 (1997).

${ }^{19}$ D.E. Aspnes and M. Cardona, Phys. Rev. B 17, 726 (1978).

${ }^{20}$ G. Platero and M. Altarelli, Phys. Rev. B 36, 6591 (1987).

${ }^{21}$ Y. Zhang, Phys. Rev. B 49, 14352 (1994).

${ }^{22}$ L.R.C. Fonseca, J.L. Jimenez, and J.P. Leburton, Phys. Rev. B 58, 9955 (1998).

${ }^{23}$ F.B. Pedersen and Y.-C. Chang, Phys. Rev. B 53, 1507 (1996).

${ }^{24}$ D.A. Broido and L.J. Sham, Phys. Rev. B 31, 888 (1985).

${ }^{25}$ Z. Ikonić, V. Milanović, and D. Tjapkin, Phys. Rev. B 46, 4285 (1992).

${ }^{26}$ C.Y. Chao and S.L. Chuang, Phys. Rev. B 46, 4110 (1992).

${ }^{27}$ L.C. Andreani, A. Pasquarello, and F. Bassani, Phys. Rev. B 36, 5887 (1987).

${ }^{28}$ G. Edwards, E.C. Valadares, and F.W. Sheard, Phys. Rev. B 50, 8493 (1994).

${ }^{29}$ P.C. Sercel and K.J. Vahala, Phys. Rev. B 42, 3690 (1990).

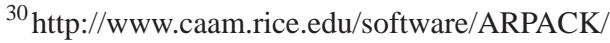

${ }^{31}$ J. Zeman, G. Martinez, K.K. Bajaj, I. Krivorotov, and K. Uchida, Appl. Phys. Lett. 77, 4335 (2000).

${ }^{32}$ D.M.-T. Kuo and Y.-C. Chang, Phys. Rev. B 61, 11051 (2000).

${ }^{33}$ O. Stier, Electronic and Optical Properties of Quantum Dots and Wires (Wissenschaft\&Technik Verlag, Berlin, 2001).

${ }^{34}$ L.W. Wang, A.J. Williamson, A. Zunger, H. Jiang, and J. Singh, Appl. Phys. Lett. 76, 339 (2000). 